\title{
Symptoms during Adolescents' First Use of Cigarettes and E-Cigarettes: A Pilot Study
}

\author{
May S. Chen ${ }^{1}$, Marissa G. Hall ${ }^{1,2}$, Humberto Parada Jr. ${ }^{2,3}$, Kathryn Peebles ${ }^{4}$, Kaitlyn E. Brodar ${ }^{1}$ \\ and Noel T. Brewer 1,2,* \\ 1 Department of Health Behavior, Gillings School of Global Public Health, University of North Carolina, \\ Chapel Hill, NC 27599, USA; maychen@email.unc.edu (M.S.C.); mghall@unc.edu (M.G.H.); \\ kbrodar@email.unc.edu (K.E.B.) \\ 2 Lineberger Comprehensive Cancer Center, University of North Carolina, Chapel Hill, NC 27599, USA; \\ hparada@live.unc.edu \\ 3 Department of Epidemiology, Gillings School of Global Public Health, University of North Carolina, \\ Chapel Hill, NC 27599, USA \\ 4 Department of Epidemiology, University of Washington Seattle; Seattle, WA 98195, USA; \\ peebles.kathryn@gmail.com \\ * Correspondence: ntb@unc.edu
}

Received: 7 August 2017; Accepted: 16 October 2017; Published: 20 October 2017

\begin{abstract}
Symptoms adolescents experience during their first time using a cigarette predict their current use, but little is known regarding the symptoms experienced during first e-cigarette use. We conducted a pilot study to understand the symptoms adolescents experience when they first tried cigarettes and e-cigarettes and the associations between these symptoms and current use. Participants were 41 adolescents in two U.S. cities who had tried cigarettes or e-cigarettes. We asked adolescents to recall the symptoms they experienced during their first cigarette or e-cigarette and categorized symptoms as negative (felt bad, coughing/chest pain, bad taste, upset stomach, dizzy/lightheaded) or positive (felt relaxed, rush/buzz). Adolescents reported fewer negative symptoms for first e-cigarette than first cigarette use (all $p<0.05$ ). Current cigarette smoking was associated with endorsing fewer negative symptoms $(\mathrm{OR}=0.49,95 \% \mathrm{CI}=[0.25,0.95])$ and more positive symptoms $(\mathrm{OR}=7.11,95 \% \mathrm{CI}=[1.47,34.33])$ at first cigarette use. First e-cigarette use symptoms were not associated with current e-cigarette use. Adolescents reported fewer negative symptoms from first e-cigarette than from first cigarette, and e-cigarette symptoms did not influence use as they do for cigarettes. Additional research is needed to confirm these findings in longitudinal studies.
\end{abstract}

Keywords: electronic cigarettes; tobacco; addiction; adolescents; smoking

\section{Introduction}

Use of e-cigarettes among U.S. adolescents is a major public health concern [1]. Although recent work has provided a better understanding of the prevalence, correlates, and health consequences of e-cigarette use [1], little is known about the symptoms that adolescents experience during their first e-cigarette and whether differences in these symptoms are associated with continued use.

Understanding the symptoms adolescents experience during their first e-cigarette is vital given prior research indicating that the symptoms individuals experience during their first cigarette are predictive of future use [2]. Positive, pleasurable sensations (e.g., relaxation, "buzz"-related sensations) during first use of cigarettes have consistently been found to increase risk for subsequent cigarette use [2-4]. Findings for negative symptoms (e.g., coughing, bad taste, upset stomach) have been mixed, 
with some studies indicating that negative symptoms discourage use $[3,5,6]$, and others demonstrating that negative symptoms increase risk for continued use [2].

Unlike conventional cigarettes, e-cigarettes work by heating a liquid solution containing nicotine and flavoring chemicals into an aerosol that is inhaled by the user [1]. Given the differences in nicotine intake methods between e-cigarettes and conventional cigarettes, it is possible that e-cigarettes may not elicit the same symptoms that adolescents typically experience during first cigarette use. Supporting this idea, two studies have found that negative symptoms were more common during first use of conventional cigarettes compared to e-cigarettes [7,8]. Furthermore, it is unclear whether initial experiences with e-cigarettes might influence likelihood of continued use. The goal of the current pilot study was to evaluate whether adolescents' initial reactions to e-cigarettes differed from their initial reactions to cigarettes, and whether these first-time experiences were associated with current smoking and e-cigarette use, respectively.

\section{Methods}

\subsection{Participants}

We conducted telephone interviews between December 2014 and September 2016 with 116 adolescents between ages 13-17 residing in North Carolina or California with a parent or guardian who participated in a randomized trial of pictorial cigarette pack warnings $[9,10]$. The parents or guardians who participated in the randomized trial were recruited through advertisements posted on social media and in retail outlets, buses, and local newspapers. All subjects gave their informed consent for inclusion before they participated in the study. The study was conducted in accordance with the Declaration of Helsinki, and the protocol was approved by the Ethics Committee of the University of North Carolina (IRB number 14-2011). A full description of the procedures appears in Peebles, et al. [10]. We excluded four adolescents from the current study due to indications that an adult had been present during the phone interview which may have influenced the adolescents' responses. Our analytic sample included only adolescents who had previously tried either cigarettes or e-cigarettes at least once. Of the 112 participants, 12 had tried cigarettes only, 12 had tried e-cigarettes, and 17 had tried both, resulting in a final sample of 41 adolescents (see Table 1 for demographic characteristics). Table 2 provides a breakdown of current use among the 41 adolescents who tried cigarettes or e-cigarettes. Sixteen percent of adolescents who tried e-cigarettes only and 33\% of adolescents who tried cigarettes only report smoking/vaping in the last 30 days. Among the 17 participants who tried both cigarettes and e-cigarettes, $76 \%$ reported being a current user: 3 currently smoked cigarettes, 3 currently used e-cigarettes only, and 7 continued to use both.

\subsection{Measures}

\subsubsection{Symptoms during First Cigarette/E-Cigarette Use}

We used open- and close-ended measures to explore the symptoms that adolescents experienced during their first time using cigarettes and e-cigarettes. Adolescents who reported that they had ever tried cigarettes or e-cigarettes were asked to describe in their own words how they felt immediately after their first time using each product. Two raters independently coded these open-ended responses as either positive, negative, or neutral. The level of inter-rater agreement was high (Cohen's $\mathrm{K}=0.85$ for responses regarding first cigarette use and $\mathrm{k}=0.87$ for responses regarding first e-cigarette use). A third rater resolved any coding discrepancies between raters.

Immediately following their open-ended responses, the survey asked adolescents whether they had experienced a set of seven symptoms during their first time smoking/vaping. These items were adapted from a previously established scale developed by DiFranzia and colleagues [4]. We coded endorsed symptoms as 1 and non-endorsed symptoms as 0 , and summed their responses to create composite scores for negative (felt bad, coughing/chest pain, bad taste in mouth, upset stomach, 
dizzy /lightheaded; range $=0-5$ ) and positive (rush/buzz, felt relaxed; range $=0-2$ ) symptoms from their first cigarette and e-cigarette use, respectively.

Table 1. Participant demographics.

\begin{tabular}{|c|c|c|}
\hline Characteristic & $\begin{array}{c}\text { Tried Cigarettes/ } \\
\text { E-Cigarettes }(n=41) n(\%)\end{array}$ & $\begin{array}{l}\text { Never Tried Cigarettes/ } \\
\text { E-Cigarettes }(n=71) n(\%)\end{array}$ \\
\hline Age, mean (SD) & $15(2)$ & $15(2)$ \\
\hline \multicolumn{3}{|l|}{ Grade } \\
\hline Middle school (grades 6-8) & $5(12)$ & $22(31)$ \\
\hline High school (grades 9-12) & $34(83)$ & $49(70)$ \\
\hline Graduated high school & $2(5)$ & $0(0)$ \\
\hline \multicolumn{3}{|l|}{ Gender } \\
\hline Male & $25(61)$ & $29(41)$ \\
\hline Female & $15(37)$ & $42(59)$ \\
\hline Transgender & $1(2)$ & $0(0)$ \\
\hline Hispanic & $3(7)$ & $11(16)$ \\
\hline \multicolumn{3}{|l|}{ Race } \\
\hline Asian & $2(5)$ & $3(4)$ \\
\hline Black or African American & $28(68)$ & $48(68)$ \\
\hline White & $8(20)$ & $15(21)$ \\
\hline Other/multiracial & $3(7)$ & $3(4)$ \\
\hline \multicolumn{3}{|l|}{ Parent education } \\
\hline High school degree or less & $14(35)$ & $19(27)$ \\
\hline Some college & $17(43)$ & $42(59)$ \\
\hline College graduate & $4(10)$ & $5(7)$ \\
\hline Graduate degree & $5(13)$ & $5(7)$ \\
\hline $\begin{array}{c}\text { Living in poverty }(<150 \% \text { of federal } \\
\text { poverty level) }\end{array}$ & $28(68)$ & $40(56)$ \\
\hline \multicolumn{3}{|l|}{ Current tobacco use (past 30 days) } \\
\hline Dual use of cigarettes and e-cigarettes & $7(17)$ & - \\
\hline Current cigarette use only & $7(17)$ & - \\
\hline Current e-cigarette use only & $5(12)$ & - \\
\hline No current use & $22(54)$ & - \\
\hline Cigarettes smoked per day, mean (SD) ${ }^{a}$ & $6(8)$ & - \\
\hline
\end{tabular}

Notes. ${ }^{\text {a }}$ Among 14 current smokers only.

Table 2. Current use among adolescents who tried e-cigarettes or cigarettes.

\begin{tabular}{|c|c|c|c|c|c|}
\hline Tobacco Use & No Current Use & $\begin{array}{c}\text { Current E-Cigarette } \\
\text { Use Only }\end{array}$ & $\begin{array}{l}\text { Current Cigarette } \\
\text { Use Only }\end{array}$ & $\begin{array}{l}\text { Current Dual } \\
\text { Use }\end{array}$ & Total \\
\hline Tried e-cigarettes only & 10 & 2 & 0 & 0 & 12 \\
\hline Tried cigarettes only & 8 & 0 & 4 & 0 & 12 \\
\hline Total & 22 & 5 & 7 & 7 & 41 \\
\hline
\end{tabular}

\subsubsection{Current Smoking and E-Cigarette Use}

The survey assessed past 30-day smoking (yes $=1$ vs. no $=0$ ) and e-cigarette use (yes $=1$ vs. no $=0$ ).

\subsection{Analytic Strategy}

We used generalized estimating equations (GEE) to compare endorsement of each of the seven symptoms from first use of cigarettes versus e-cigarettes. This analytic strategy adjusts standard errors for correlated data from repeated measurements within individuals (some adolescents had tried 
both cigarettes and e-cigarettes) [11]. We used logistic regression to examine associations between the summed number of positive or negative symptoms endorsed from first use and current cigarette and e-cigarette use. Models adjusted for adolescents' age and parent/guardian condition assignment in the parent study. We used a critical alpha of 0.05 and SAS version 9.4 (SAS Institute Inc., Cary, NC, USA) for analyses.

\section{Results}

\subsection{Open-Ended Reports of Symptoms from First Use}

Of the 29 adolescents who had tried conventional cigarettes, $22(76 \%)$ reported experiencing negative symptoms only, $2(7 \%)$ reported feeling neutral only, and $4(14 \%)$ reported experiencing both positive and negative symptoms. No participants reported positive symptoms only. The negative symptoms that adolescents reported included feeling dizzy, sick, bad taste in their mouth, difficulty breathing, and headache (Table 3). In contrast, of the 29 adolescents who had tried e-cigarettes, $9(31 \%)$ reported experiencing negative symptoms only, 12 (41\%) reported feeling neutral only, 6 (21\%) reported experiencing positive symptoms only, and $2(7 \%)$ reported experiencing both positive and negative symptoms. Twenty-five of the 29 adolescents (86\%) reported that they felt "normal", "no change", or "the same" after their first e-cigarette. Three (18\%) of the 17 adolescents who had tried both products made unprompted comparisons of the symptoms they experienced during their first use of e-cigarettes and conventional cigarettes. These participants stated, "I got my nicotine rush, but without the bad taste and bad smell. Nicotine rush, nothing else different", and that e-cigarettes have "all of the good effects of a cigarette, but doesn't upset my stomach and doesn't make my mouth taste weird". In addition, one participant stated that the flavoring chemicals mask the negative symptoms: "there weren't any side effects because it was a vape pen and it had a taste. I just tasted the flavor".

Table 3. Example open-ended responses about first experiences with cigarettes and e-cigarettes.

\begin{tabular}{|c|c|c|c|}
\hline Tobacco Product & Negative & Neutral & Positive \\
\hline Cigarettes & $\begin{array}{l}\text { - } \quad \text { Horrible. It was gross and I } \\
\text { had a weird taste in my } \\
\text { mouth. It made me feel sick, } \\
\text { like I never wanted to } \\
\text { do it again." } \\
\text { " } \quad \text { Disgusting, my head was } \\
\text { hurting, light-headed. It } \\
\text { really wasn't a good feeling." } \\
\text { "I don't like it cause it made } \\
\text { me feel weird, it made it hard } \\
\text { to breathe ... I didn't like it." } \\
\text { "Dizzy. I wasn't happy about } \\
\text { it, I didn't see what the hype } \\
\text { was. Yuck." } \\
\text { "My palms were sweaty and } \\
\text { I felt shaky and dizzy." }\end{array}$ & $\begin{array}{l}\text { - "Kinda like "eh"." } \\
\text { - "I don't know, I kinda felt } \\
\text { the same." } \\
\text { - "Fine" }\end{array}$ & $\begin{array}{l}\text { - } \quad \text { "Uplifted" } \\
\text { - } \quad \text { "Somewhat relieved. Like } \\
\text { - } \quad \text { relieved of stress." } \\
\text { "Cool" }\end{array}$ \\
\hline E-cigarettes & $\begin{array}{l}\text { - “Um, I didn't like it, so I } \\
\text { - } \quad \text { "Gover did it again." } \\
\text { rush. Hurt my thro bit a head } \\
\text { - "Kinda grossed out. Wasn't } \\
\text { my thing." }\end{array}$ & $\begin{array}{l}\text { - } \quad \text { "I didn't feel any different. } \\
\text { I didn't really feel } \\
\text { anything." } \\
\text { " } \quad \text { There weren't any side } \\
\text { effects because it was a } \\
\text { vape pen and it had a taste. } \\
\text { I just tasted the flavor. I felt } \\
\text { kind of normal." } \\
\text { "I felt all right, I guess. } \\
\text { - The same." } \\
\text { "It didn't really make me } \\
\text { feel any type of way. I } \\
\text { felt normal." }\end{array}$ & $\begin{array}{l}\text { - } \quad \text { "Good. Like, nothing different, } \\
\text { but just felt better. Confident." } \\
\text { "It has all of the good effects of } \\
\text { a cigarette but doesn't upset } \\
\text { my stomach and doesn't make } \\
\text { my mouth taste weird." } \\
\text { "I got my nicotine rush, but } \\
\text { without the bad taste and bad } \\
\text { smell. Nicotine rush, nothing } \\
\text { else different." } \\
\text { "Made me feel a little calm } \\
\text { because of the flavor." }\end{array}$ \\
\hline
\end{tabular}




\subsection{Quantitative Comparison of Symptom Endorsement from First Use}

Adolescents were more likely to endorse each of the five negative symptoms (felt bad; coughing, chest pain, and irritated eyes; bad taste; upset stomach; dizzy or lightheaded) when they first tried conventional cigarettes compared to e-cigarettes (Figure 1, all $p<0.05$ ). For example, 59\% of adolescents endorsed coughing, chest pain, or irritated eyes after their first cigarette compared to $17 \%$ of adolescents for e-cigarettes, and $41 \%$ of adolescents endorsed having an upset stomach after their first cigarette, compared to only $7 \%$ of adolescents for e-cigarettes. Adolescents were also more likely to endorse getting a rush or buzzed feeling from cigarettes than from using e-cigarettes $(62 \%$ vs. $21 \% ; p<0.05)$.

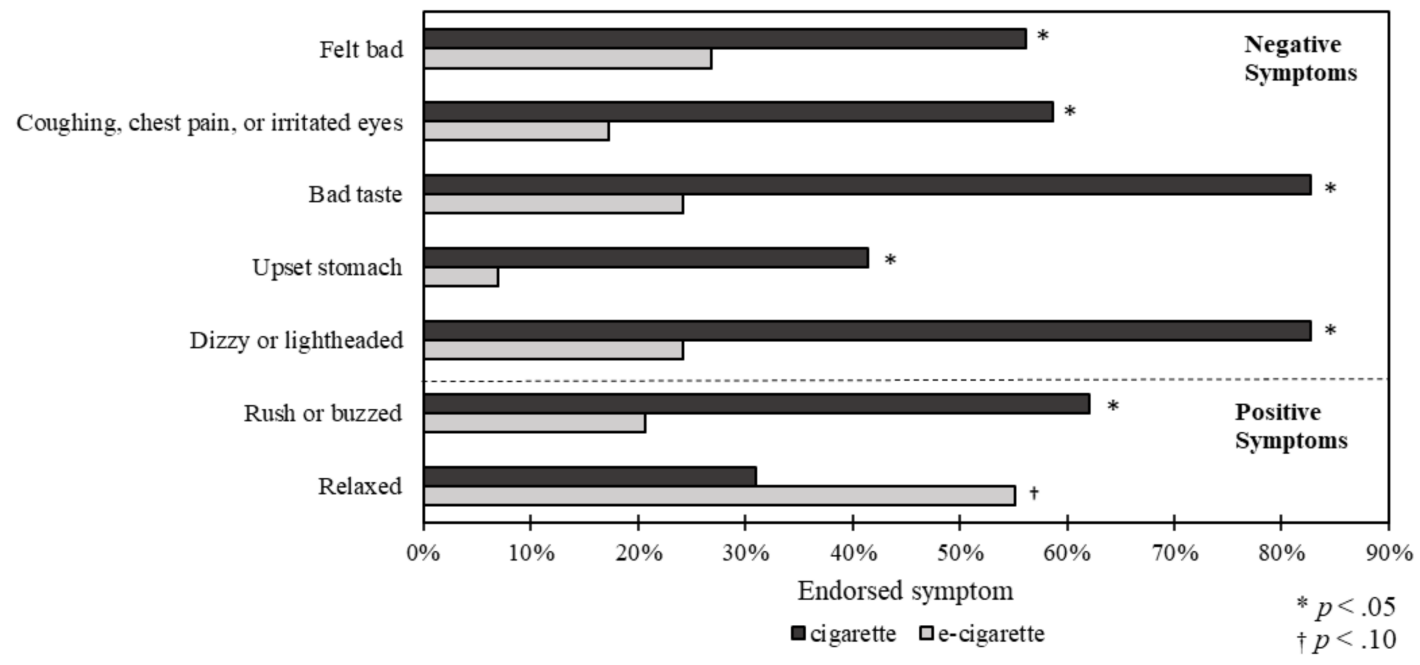

Figure 1. Symptoms during first use of cigarette vs. e-cigarette $(n=41)$.

\subsection{Association between Symptoms and Current Use}

Table 4 presents associations between initial symptoms and current cigarette and e-cigarette use in the past 30 days. Endorsement of more negative symptoms during first cigarette use was associated with lower odds of current smoking among the 29 adolescents who had tried cigarettes ( $O R=0.49,95 \%$ $\mathrm{CI}=[0.25,0.95])$. Endorsement of more positive symptoms was associated with greater odds of current smoking $(\mathrm{OR}=7.11,95 \% \mathrm{CI}=[1.49,34.33])$. Among the 29 adolescents who had tried e-cigarettes, neither positive nor negative symptoms during first e-cigarette use were associated with current e-cigarette use (negative symptoms $\mathrm{OR}=0.93,95 \% \mathrm{CI}=[0.50,1.72]$; positive symptoms $\mathrm{OR}=1.73,95 \%$ $\mathrm{CI}=[0.50,5.92])$.

Table 4. Associations between symptoms during first use and current tobacco use.

\begin{tabular}{ccccc}
\hline Predictor & \multicolumn{2}{c}{ Cigarette Use $(\boldsymbol{n}=\mathbf{2 9 )}$} & \multicolumn{2}{c}{ E-cigarette Use $(\boldsymbol{n}=\mathbf{2 9 )}$} \\
\hline & OR & $95 \%$ CI & OR & $95 \%$ CI \\
Number of negative symptoms & 0.49 & $0.25-0.95$ & 0.93 & $0.50-1.72$ \\
Number of positive symptoms & 7.11 & $1.49-34.33$ & 1.73 & $0.50-5.92$ \\
\hline
\end{tabular}

Note. Analyses control for adolescent age and parents' assigned trial condition.

\section{Discussion}

E-cigarettes appear to be less off-putting than conventional cigarettes, which may contribute to their growing popularity among adolescents. Adolescents in two U.S. cities reported experiencing fewer negative symptoms from e-cigarettes than conventional cigarettes in our small pilot study, findings that are consistent with previous studies of a national sample of adults [7] and of adolescents in Texas [8]. A novelty of our study is the use of open-ended questions, in which adolescents made unprompted comments about experiencing fewer negative symptoms from e-cigarettes. 
A natural question is what association these recalled symptoms may have with current tobacco use. Adolescents who recalled more positive symptoms during their first time using cigarettes were more likely to be current smokers, and adolescents who endorsed more negative symptoms were less likely to be current smokers. Other studies have reported similar findings among adults and adolescents [2-4], and our findings support health behavior models which suggest that a positive first experience with cigarettes would predict continued use while a negative first experience would discourage use [2,5].

In contrast, symptoms experienced at first e-cigarette use were not associated with current e-cigarette use. In the only other study to examine the influence of adolescents' first experiences with e-cigarettes on current use, Mantey et al. also found that positive and negative initial experiences at first use had no effect on past 30-day e-cigarette use [8]. Together, these findings suggest that the mechanisms leading to regular use of e-cigarettes and cigarettes among adolescents may have important differences. It is possible that initial symptoms to e-cigarettes do not leave a strong initial impression on adolescents, and that other factors not measured in the current study (e.g., novelty, accessibility, social norms) are more salient predictors of current e-cigarette use. Given our small cross-sectional study design, we encourage caution when interpreting our results. Future studies should consider replicating these results with larger, probability-based samples and with longitudinal data to establish the temporality of associations. Given that e-cigarette devices vary widely in quality and design [1], future studies should also consider how choice of e-cigarette device used might influence symptoms experienced and likelihood of later use. We also did not collect data on whether adolescents who had tried both products had tried cigarettes or e-cigarettes first; future studies should consider the temporal order of adolescents' first experiences with cigarettes and e-cigarettes. Finally, because the open-ended questions were embedded within a longer quantitative survey, we were unable to probe further on why adolescents stopped or continued smoking after their initial experience. Additional qualitative work is needed to examine why positive and negative initial symptoms are linked to current use for cigarettes but not for e-cigarettes.

Despite having several limitations, our pilot study provides a novel contribution to the literature. We build on previous work by demonstrating the generalizability of the relationships between initial symptoms and current use to a high risk sample of adolescents (e.g., children of smokers and majority low-income sample). In addition, the use of both open- and close-ended data allowed us to develop a better understanding of the symptoms that adolescents experience during first use of e-cigarettes compared to cigarettes. Future quantitative work is needed examining the temporal sequencing of first experiences with e-cigarettes and cigarettes, the degree to which adolescents experience particular positive or negative symptoms, and whether experiencing specific clusters of symptoms may confer greater risk for future use.

\section{Conclusions}

In sum, our findings underscore the potential appeal of e-cigarettes during adolescents' first use compared to traditional cigarettes, and the value of further exploration in this emerging area. Adolescence is a critical developmental period when experimentation with tobacco products typically begins [1]. Thus, gaining a better understanding of how their initial experiences with cigarettes and e-cigarettes might shape later use and developing public health interventions that target these effects are important future steps for preventing tobacco use among this key population.

Acknowledgments: Research reported in this publication was supported by the National Cancer Institute of the National Institutes of Health under Award Number P50CA180907. F31CA196037 and T32-CA057726 from the National Cancer Institute of the National Institutes of Health supported MGH's time writing the paper. The content is solely the responsibility of the authors and does not necessarily represent the official views of the National Institutes of Health.

Author Contributions: May S. Chen, Humberto Parada Jr., Marissa G. Hall, and Noel T. Brewer developed the study and analysis plan; Kathryn Peebles collected the data; May S. Chen, Humberto Parada Jr., and Marissa G. Hall conducted the analysis; May S. Chen drafted the manuscript; Humberto Parada Jr., 
Marissa G. Hall, Kaitlyn E. Brodar, Kathryn Peebles, and Noel T. Brewer contributed to the interpretation of the results and provided substantive edits to the manuscript. All authors read and approved the final manuscript.

Conflicts of Interest: Noel T. Brewer has served as a paid expert consultant in litigation against tobacco companies. The other authors declare no conflicts of interest.

\section{References}

1. U.S. Department of Health and Human Services. E-Cigarette Use among Youth and Young Adults: A Report of the Surgeon General; Office on Smoking and Health, National Center for Chronic Disease Prevention and Health Promotion, Centers for Disease Control and Prevention, U.S. Department of Health and Human Services: Atlanta, GA, USA, 2016.

2. DiFranza, J.R.; Savageau, J.A.; Fletcher, K.; Ockene, J.K.; Rigotti, N.A.; McNeill, A.D.; Coleman, M.; Wood, C. Recollections and repercussions of the first inhaled cigarette. Addict. Behav. 2004, 29, 261-272. [CrossRef] [PubMed]

3. Richardson, C.G.; Okoli, C.T.C.; Ratner, P.A.; Johnson, J.L. Empirical support for a multi-dimensional model of sensations experienced by youth during their initial smoking episodes. Addiction 2010, 105, 1827-1834. [CrossRef] [PubMed]

4. Wellman, R.J.; DiFranza, J.R.; O'Loughlin, J. Recalled first reactions to inhaling nicotine predict the level of physical dependence. Drug Alcohol Depend. 2014, 143, 167-172. [CrossRef] [PubMed]

5. Leventhal, H.; Diefenbach, M.; Leventhal, E.A. Illness cognition: Using common sense to understand treatment adherence and affect cognition interactions. Cognit. Ther. Res. 1992, 16, 143-163. [CrossRef]

6. Rodriguez, D.; Audrain-McGovern, J. Construct validity analysis of the early smoking experience questionnaire for adolescents. Addict. Behav. 2004, 29, 1053-1057. [CrossRef] [PubMed]

7. Wackowski, O.A.; Bover Manderski, M.T.; Delnevo, C.D.; Giovenco, D.P.; Lewis, M.J. Smokers' Early e-cigarette experiences, reasons for use and use intentions. Tob. Regul. Sci. 2016, 2, 133-145. [CrossRef] [PubMed]

8. Mantey, D.S.; Harrell, M.B.; Case, K.; Crook, B.; Kelder, S.H.; Perry, C.L. Subjective experiences at first use of cigarette, e-cigarettes, hookah, and cigar products among Texas adolescents. Drug Alcohol Depend. 2017, 173, 10-16. [CrossRef] [PubMed]

9. Brewer, N.T.; Hall, M.G.; Noar, S.M.; Parada, H.; Stein-Seroussi, A.; Bach, L.E.; Hanley, S.; Ribisl, K.M. Effect of pictorial cigarette pack warnings on changes in smoking behavior: A randomized clinical trial. JAMA Intern. Med. 2016, 176, 1-8. [CrossRef] [PubMed]

10. Peebles, K.; Hall, M.G.; Pepper, J.K.; Byron, M.J.; Noar, S.M.; Brewer, N.T. Adolescents' responses to pictorial warnings on their parents' cigarette packs. J. Adolesc. Health 2016, 59, 635-641. [CrossRef] [PubMed]

11. Hanley, J.A.; Negassa, A.; Forrester, J.E. Statistical analysis of correlated data using generalized estimating equations: An orientation. Amer. J. Epidemiol. 2003, 157, 364-375. [CrossRef]

(C) 2017 by the authors. Licensee MDPI, Basel, Switzerland. This article is an open access article distributed under the terms and conditions of the Creative Commons Attribution (CC BY) license (http:/ / creativecommons.org/licenses/by/4.0/). 